\title{
Early Stages in the Growth of Electric Field-Induced Surface Fluctuations
}

\author{
K. Amanda Leach, Zhiqun Lin, ${ }^{\dagger}$ and Thomas P. Russell* \\ Department of Polymer Science and Engineering, University of Massachusetts at Amherst, \\ Amherst, Massachusetts 01002
}

Received September 8, 2004; Revised Manuscript Received March 17, 2005

\begin{abstract}
Real-time measurements of fluctuations arising from electrohydrodynamic instabilities at polymer liquid/air interfaces were used to characterize the selection of a dominant wavelength and the rate at which this fluctuation grows. Reflection interference fringes from the film surface were used to measure the growth rates of fluctuations and their dependence on electrostatic pressure. The growth rate was found to exponentially depend on time and strongly depend on the applied voltage and initial distance between the liquid surface and the opposing electrode. Results at early times are in quantitative agreement with predictions from a linear stability analysis, yet deviations are seen with increasing time and define the limits over which the linearized theory is applicable.
\end{abstract}

\section{Introduction}

The stability of thin polymer films and control of structure and morphology in thin films are essential for the practical use of polymers in many applications. The static and dynamic aspects of thin film instabilities arising from polymer/substrate and polymer/air interactions, that result in characteristic dewetting patterns, have been thoroughly studied. ${ }^{1}$ The influence of external electrostatic forces on the stability of a polymer surface has also been studied in detail, both experimentally and theoretically. The use of electrostatic pressure to destabilize polymer surfaces underpins electrospinning technology, by which submicron diameter polymer fibers can be readily produced. Studies have also appeared on the dynamics of deformation of a polymer surface by an electric field, when free charges are present at the surface. $^{2-5}$ Such experiments are challenging, not only because of the high voltages required but also because of the small length scales and extremely short time scales.

The effect of an applied electric field on thin polymer films, in the absence of free charges, is an area of active research. Schaffer et al. described electrohydrodynamic instabilities in polystyrene (PS), poly(methyl methacrylate) (PMMA), and poly(1-bromostyrene) (PSBr) films between parallel electrodes such that an air gap is left between the polymer film and opposite electrode. ${ }^{6,7}$ Their results showed that changing the magnitude of the electric field offers a simple way to control the length scale of structures formed by a balance between the electrostatic pressure and the Laplace pressure governed at the polymer/air interface. When electrostatic pressure overcomes surface tension, fluctuations of a specific wavelength are amplified, grow, and eventually span the electrodes, forming columns of polymer with a characteristic center-to-center distance. Under a patterned electrode, the polymer film will replicate either the topographic patterning or chemical patterning of the electrodes down to a length scale of $\sim 140 \mathrm{~nm}$.

Chou and Zhuang developed a process, termed lithographically induced self-assembly (LISA), where similar

\footnotetext{
† Current address: Department of Materials Science and Engineering, Iowa State University, Ames, IA 50011.
}

arrays of columns in PMMA films mounted between silicon wafers with an air gap can be produced.8,9 Although no external field was applied, a qualitative dependence of column diameter and period on molecular weight was reported. Using this process, arrays of columns were produced under topographically patterned surfaces having triangular, rectangular, and square features. The quality of the lateral ordering of the columns depended on the commensurability of characteristic spacing of the columns with the lateral pattern dimension. They suggested that image charges in the mask gave rise to a local electric field that caused structure formation. Subsequently, Deshpande et al. ${ }^{10}$ made in situ optical observations of LISA which showed that the initial formation of columns occurs at the corners of the pattern, then along the edges, and, finally, moving to the interior of the pattern.

Lin et al. ${ }^{11}$ theoretically extended the model of Schaffer et al. ${ }^{6,7}$ to the polymer-polymer bilayer case and experimentally to bilayer systems of oligomeric dimethylsiloxane (ODMS), poly(isoprene) (PI), and oligomeric styrene (OS). They showed that by filling the air gap with another liquid the interfacial tension decreased and the characteristic spacing decreased, in reasonable agreement with the decrease predicted from calculations using the extended theory. Further experiments demonstrated that the extended theory captured the important experimental parameters so well that the bilayer and single layer data could be collapsed onto a single master curve using reduced variables. ${ }^{12}$ The agreement between the experimentally measured size scale and those predicted analytically was excellent. However, they could not explain the observed 50-fold decrease in time required to form columns. Furthermore, Pease and Russel $^{13}$ later compared experimental data available from Lin et al., ${ }^{11,12}$ Schaffer et al., ${ }^{6}$ and Chou et al. ${ }^{8}$ for both single polymer and bilayer systems and found that, in all but one case, the characteristic times predicted by theoretical models was much shorter than the reported experimental time.

Structure formation at a polymer-air interface in an electric field is analogous to phase separation in polymer blends. In the latter system, phase domains develop over time with a periodicity equal to the dominant wave- 
Table 1. Physical Properties of PDMS Materials Studied

\begin{tabular}{cccc}
\hline $\begin{array}{c}\text { viscosity from } \\
\text { manufacturer }(\mathrm{cSt})\end{array}$ & $\begin{array}{c}\text { viscosity by } \\
\text { rheometry }(\mathrm{Pa} \mathrm{s})\end{array}$ & $\begin{array}{c}\text { weight-averaged } \\
\text { mol wt }(\mathrm{g} / \mathrm{mol})\end{array}$ & PDI \\
\hline 500 & 0.494 & 36000 & 1.4 \\
1000 & 1.071 & 67000 & 1.8 \\
10000 & 10.25 & 146000 & 2.0
\end{tabular}

length of concentration fluctuations in the system. The wavelength of these fluctuations is dictated by a balance between thermodynamics and kinetics. Thermodynamics, which is governed by the interfacial energy resulting from the formation of phases, favors the growth of large domains, while diffusion kinetics favors the growth of smaller phases. The kinetics of phase separation in polymer blends, i.e., the rate at which fluctuations grow at the early stages of the phase separation, is characterized by an exponential growth, in accordance with the linearized Cahn-Hilliard arguments. However, at later stages, deviations from this behavior are seen, and hydrodynamics associated with the flow of the polymers must be considered. The growth of fluctuations at the air-polymer interface in an electric field should follow a similar pattern, as it is similarly governed by a balance between surface tension and electrohydrodynamic flow. The linearized theory of Schaffer et al. ${ }^{6,7}$ predicts that the rate of growth in the amplitude of the dominant wavelength should be exponential. However, deviations from this should be expected as the flow of the polymer in the thin films becomes dominant.

Here, the early and intermediate stages of the growth of electrohydrodynamic instabilities in PDMS thin films are discussed. Using in situ laser scanning confocal microscopy, reflection interference fringes arising from film thickness fluctuations are investigated as a function
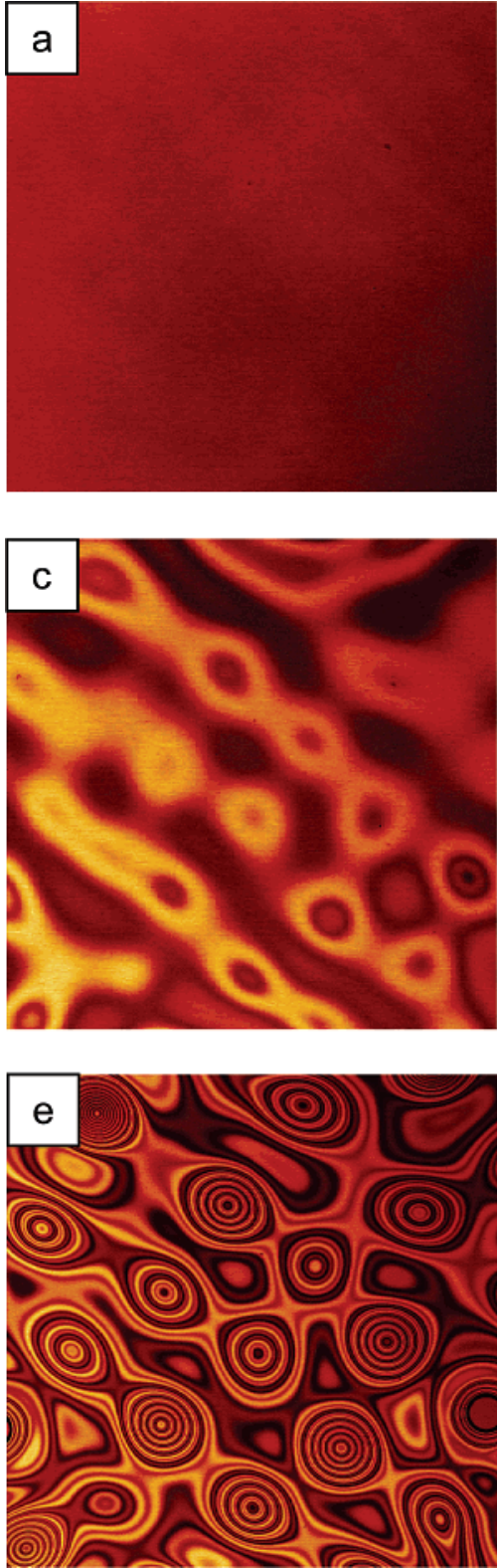
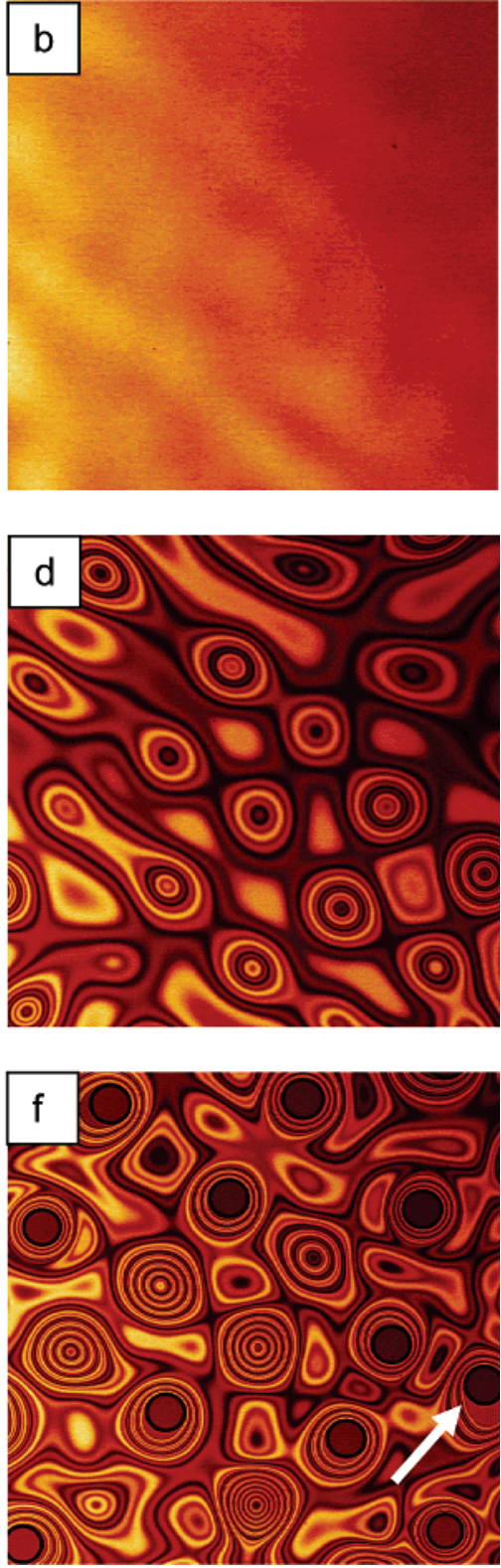

Figure 1. Various stages of structure development by electric field-amplified instability, as a function of time, of a PDMS film on silicon. (a) $[0.0 \mathrm{~s}]$ The film is initially featureless. (b) [36.6 s] Fluctuations appear as lateral variations in intensity over the plan of the surface. (c) [75.8 s] Field-amplified peaks exhibit reflection interference fringes as height increases. (d) [109.7 s] Peaks are encircled by fringes as height increases further. (e) [129.3 s] As peaks grow, the number of fringes around each peak increases. (f) $[144.1 \mathrm{~s}]$ When peaks span the two planar electrodes, cylindrical structures are formed. The arrow in (f) indicates a pillar which has shifted laterally after electrode contact. Laser scanning confocal micrographs were acquired by reflection imaging through a transparent electrode. Laser wavelength is $458 \mathrm{~nm}$; image dimension is $740 \times 740 \mu \mathrm{m}^{2}$. 
of time, before columns are formed between the electrodes. Quantitative information on the growth rates of fluctuations was obtained that delineates a temporal regime in which the linearized theoretical arguments are applicable.

\section{Experimental Section}

Linear poly(dimethysiloxane) fluids were spin-coated from $20 \%(\mathrm{w} / \mathrm{w}$ ) solutions in toluene or heptane, onto doped silicon wafers. Three PDMS viscosities were used: 500, 1000, and $10000 \mathrm{cSt}$, as specified by the manufacturer. GPC and rheometry measurements gave the molecular weights and zero shear rate viscosities, as shown in Table 1 . The dielectric constants of these fluids at $20^{\circ} \mathrm{C}$ were measured to be 3.0 over a frequency range of $5 \mathrm{~Hz}-100 \mathrm{kHz},{ }^{14}$ using a broadband impedance spectrometer (Novocontrol) equipped with a liquid cell (BDS 1308). Indium tin oxide (ITO)-coated glass slides (Delta Technologies, Ltd.) were mounted opposite the films, with a spacer of evaporated silicon oxide or with polyimide films (Mictron, Toray Industries) separating the silicon substrate and glass slide, ensuring precise air gaps of $3-10 \mu \mathrm{m}$. The fill fraction, i.e., the ratio of film thickness to distance between silicon and glass, was varied from 0.1 to 0.5 . Voltages ranging from 40 to $90 \mathrm{~V}$ were applied from a dc power supply to the silicon wafer and ITO glass. A Filmetrics spectral reflectance instrument was used to measure film and gap thickness. A confocal laser scanning microscope (Leica TCSSP2) was used to record reflection images of the film at a wavelength of 458 or $543 \mathrm{~nm}$.

In previous experiments, we and others ${ }^{10,14}$ observed that structure growth is perturbed at the edges of the film, especially when the gap is smaller at one edge than another, creating a lateral gradient in electrostatic pressure. Careful attention was paid to the mounting of the ITO glass to make the electrodes as parallel as possible. The air gap was measured after mounting by spectral reflectance measurements, and in situ observations were made at the same spot where the gap measurement was made. Even without a wedgeshaped gap, structure development may be affected by curvature of the electric field lines at the film's edge, so only regions near the center of the film were investigated. Perturbations arising from heterogeneities, such as dust particles in the film, also produce significant effects on structure formation. Sample preparation was therefore performed to minimize contaminants. Glass electrodes were cleaned by ultrasonication in aqueous ethanolamine solution, silicon wafers were cleaned in sulfuric acid bath containing inorganic oxidizers, and polymer solutions were filtered using $0.2 \mu \mathrm{m}$ PTFE filters.

\section{Results and Discussion}

When the polymer film is above its glass transition temperature, a spectrum of capillary waves is present at the liquid-air interface due to thermal fluctuations. However, surface tension suppresses the amplification of these waves. Therefore, as shown by the interference optical micrograph in Figure 1a, the film surface is initially featureless. Slight variations in the intensity over the field of view are possible due to positioning of the sample at a slight angle to the imaging plane. If there is a significant gradient in gap spacing, i.e., the gap is wedge-shaped, another set of fringes, parallel lines running perpendicular to the gradient, will be evident in the micrograph even when the film is smooth. In the experiments no fluctuations were observed in the absence of an applied electric field, implying that PDMS behaves as a perfect dielectric.

When a voltage is applied between the substrate and opposite electrode, electrostatic pressure acts against the surface tension. If there is sufficient voltage and dielectric contrast at the interface, electrostatic pressure overcomes surface tension and causes a roughening of
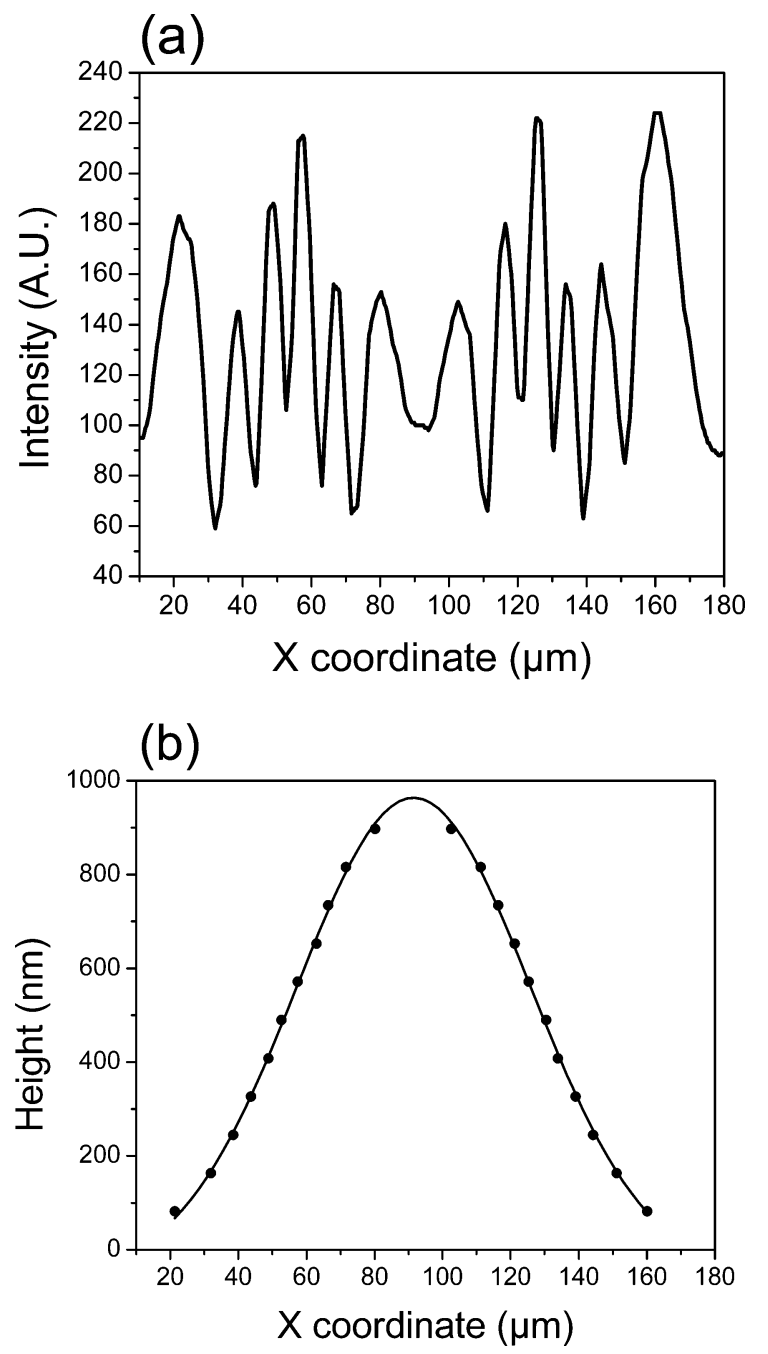

Figure 2. (a) Cross-sectional intensity profile of a peak showing interference fringes. (b) Calculation of peak shape from fringe spacing (squares) and Gaussian fit to points (red line).

the film due to the amplification of surface waves. It should be noted that, rather than a pulsed laser, a continuous wave laser was employed to raster-scan the sample. Therefore, no heat is expected to be generated across the sample. In other words, no temperature gradient-induced instabilities would be expected. ${ }^{15}$ Within seconds of application of electrostatic pressure to the PDMS-air interface, a lateral pattern of intensity peaks is apparent in the confocal image, as shown in Figure 1b. The reflective interference of light is dependent on the refractive index of the film, $n$, and the incident wavelength, $\lambda$, such that the change in height, $\Delta h$, between intensity maxima or minima is given by $\Delta h=$ $\lambda / 2 n$. For these experiments, $\Delta h$ ranges from 160 to 200 $\mathrm{nm}$. Thus, as intensity increases from a minimum to a maximum in Figure 1b, thickness variations are less than $\Delta h / 2$, i.e., less than $80 \mathrm{~nm}$. The fluctuations have a typical spacing of $100 \mu \mathrm{m}$ or more in the plane of the film. Consequently, these fluctuations represent only minor height variations of the film surface and, as such, conform to the linearized theoretical framework which assumes the lubrication approximation.

The amplitude of height variations increases with time due to the electrostatic pressure. The intensity of bright regions of Figure $1 b$ is increased in Figure 1c. However, in some areas the center of the bright spot 


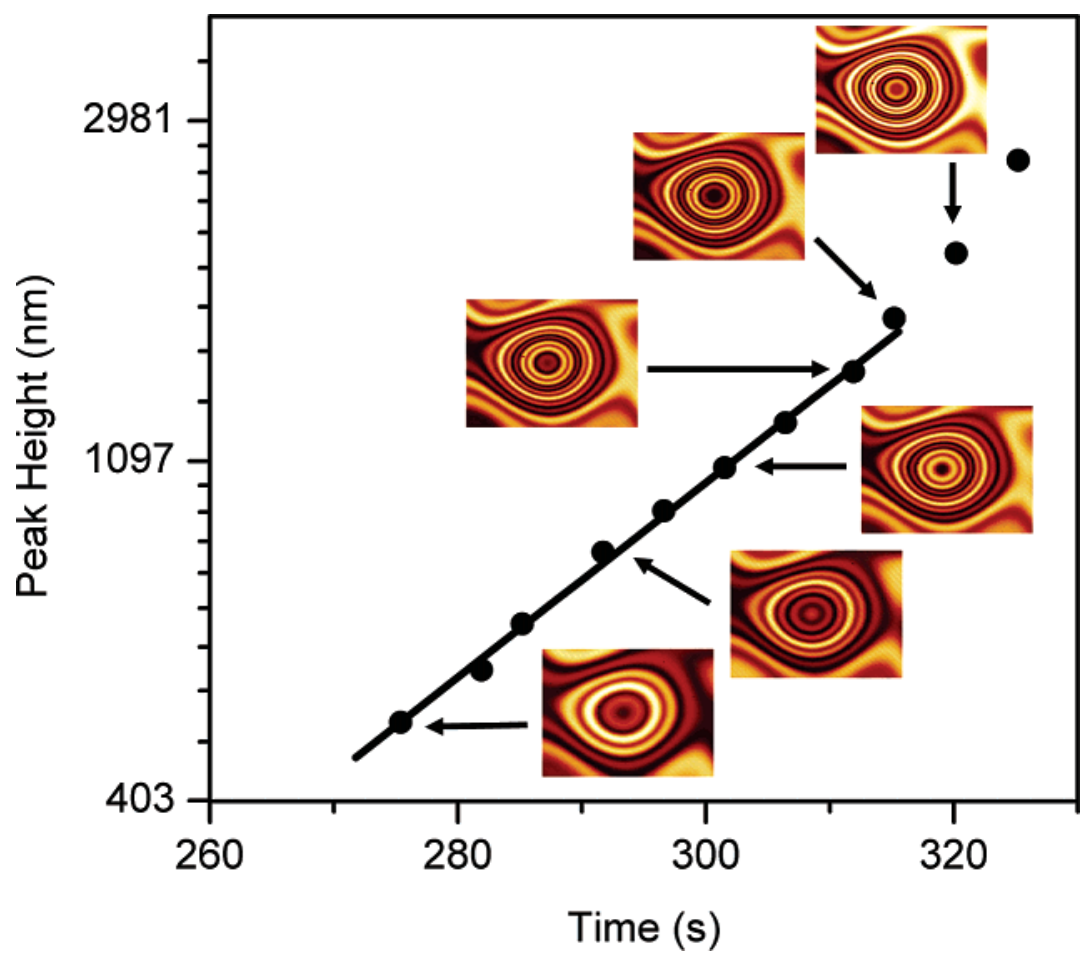

Figure 3. Development of peak height (natural log scale) with time for a typical feature. Line indicates best fit for data up to $320 \mathrm{~s}$. Image dimension is $198 \times 384 \mu \mathrm{m}^{2}$.

becomes darkened. Here, a decrease in intensity results from destructive interference of reflected light, as the height approaches a thickness for which an intensity minimum is observed. Comparison of parts $\mathrm{c}-\mathrm{f}$ of Figure 1 , in which the final column morphology is shown, confirms that the characteristic spacing of the final columnar morphology corresponds to the wavelength of the undulations observed at early times.

As the peak height continues to increase with time, the undulations in the film surface are characterized by rings of interference fringes, as shown in Figure 1d. At the base of each feature, the fringes are noticeably broader and less axially symmetric than at the center due to the sensitivity of the position of the peak to flow in the plane of the film, resulting from the mass transport from the surrounding flat film, and the smooth peak shape arising from the Laplace pressure normal to the film surface. This micrograph illustrates that the characteristic distance between growing peaks is determined by the competition between electrostatic and Laplace pressures. For example, in the lower left quadrant of Figure 1d, a row of three peaks is visible, of which the middle peak eventually decreases in height while the other two peaks grow, the result of which is shown in Figure 1e. Figure 1d also shows that disparities in height between peaks which, though slight at first, become amplified with time as a result of the exponentially increasing growth rate.

When the amplitude of the fluctuations increases sufficiently to span the air gap between the film and upper electrode, the polymer fluid and the electrode come into contact. First, contact is made by the center of the peak, followed by an equilibration of the structure to a columnar shape. In Figure 1f, the columns of PDMS appear as circular contacts between the film and the upper electrode. There is a strong driving force toward the alignment of air-polymer surfaces parallel to the electric field to minimize the electrostatic pressure. Furthermore, spreading of PDMS on the ITO glass results in a reduction of surface free energy at the upper electrode.

The thermodynamic factors of electrostatic pressure and surface energy minimization favor the coalescence of columns into one larger structure as the equilibrium state of this system. This was never observed, though coalescence of the columns was observed in some PDMS/ air systems. Columns can coalesce as they grow in radius by drawing additional material from the fluid reservoir on the lower electrode. Similar experiments with other polymers show this behavior only at high temperature. ${ }^{14}$ Pattern replication occurs precisely by this coalescence mechanism, as shown by Lin. ${ }^{14}$

The growth in amplitude of the surface waves was determined from the change in the film thickness determined from the interference fringes that encircle each peak. Since the period of the fringes is dictated by the slope of the film, they can be used to calculate the three-dimensional shape of the surface fluctuations. Figure 2 shows an example of a surface topography cross section calculated from interference fringes. The peak height was determined by fitting the resulting topographic profile to a Gaussian peak function, weighted with strictest fitting constraints at the center of the peak, so as to most accurately estimate the height in the center of the feature. Though not predicted theoretically, the Gaussian peak shape was found to suitably describe the shape of the surface features at early times.

From a frame-by-frame calculation of peak height, the growth in the amplitude of the waves was found to depend exponentially on time. A typical growth curve is shown in Figure 3 in a semilog plot. A single exponent could be used to describe the early stages of structure growth. However, the growth in some cases was found to accelerate toward the upper electrode at the final stages of structure growth, and the rate became faster than the initial exponential dependence. The linearized theory takes into account the increased electrostatic pressure at the peaks, which is the reason for the initial 


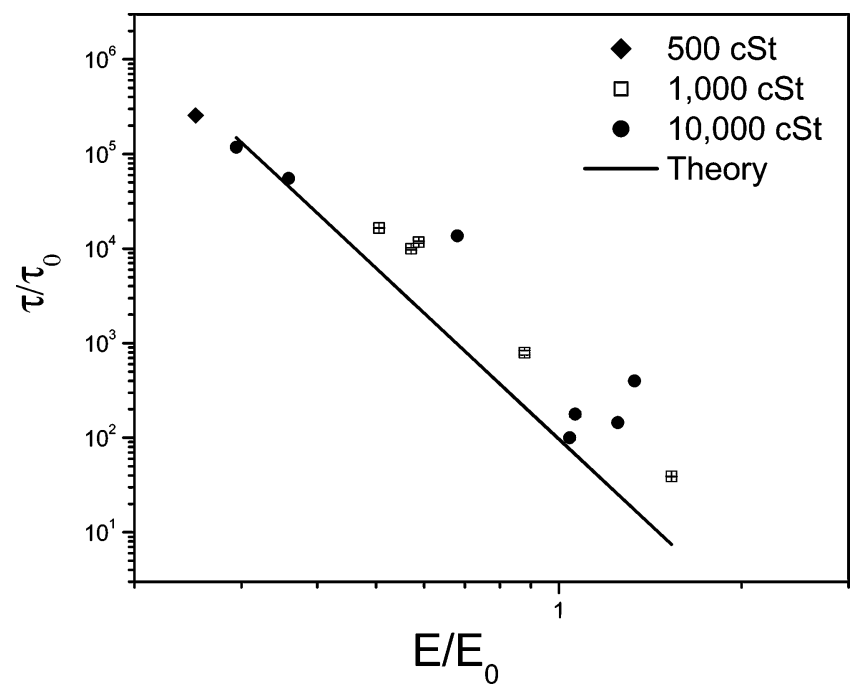

Figure 4. Variation of dimensionless characteristic time with dimensionless electric field and comparison with predicted values. Both axes have logarithmic scales.

stages of exponential growth. In the later stages, however, the shape of the fluctuations could not be described by a simple smooth function since they became increasingly pointed. The electric field at the peak is higher than elsewhere across the surface, and field lines are no longer parallel. The peak shape at these late stages could not be measured directly due to increasingly rapid changes in shape. In the final stages of pillar formation, the shape more closely resembles an electrohydrodynamic spout, seen by Oddershede and Nagel, ${ }^{5}$ where a divergence in tip curvature was found as the liquid was drawn to the opposing electrode.

In all experiments, when the amplified waves grew and spanned the air gap to the opposite electrode, an array of polymer columns formed, where the center-tocenter distance of the columns was identical to the lateral distance between the most rapidly growing fluctuations. However, not all of the fluctuations grew into columns simultaneously. Rather, slight differences in the initial height of the peaks were enhanced due to the exponential nature of the growth rate. As shown in Figure 1, the sequence in which pillars form seems to be stochastic. In some cases, the final formation of a pillar caused draining of the underlying "reservoir" of material and, therefore, slowed the growth of adjacent features. This type of dynamic effect is not incorporated into the linear stability model and resulted in deviations from predicted growth rates. Analysis of characteristic growth rates, therefore, focused on the fastest growing peaks, which are least perturbed by such effects, that could be described by a simple Gaussian function.

The experimental data consistently show an exponential growth of the peak height with time (Figure 3). For thinner films and at lower fields, the exponent is smaller, corresponding to slower structure formation. For thicker films and higher fields, the exponent was larger, corresponding to faster structure formation. In the linearized stability analysis, the wavelike fluctuations in the film surface were modeled as changes in height, $h$, as a function of spatial coordinate, $x$, and time, $t$, along with parameters of amplitude, $u$, wavenumber, $q$, and growth time scale, $\tau: h(x, t)=h_{0}+u \mathrm{e}^{i q x+t / \tau}$. It is the characteristic growth rate, $\tau^{-1}$, which determines the slope of the line fit to data at early times on semilog axes, as represented in Figure 3. To enable comparison between experiments, the electric field strength in the polymer, $E_{\mathrm{P}}$, for each experiment was reduced to a dimensionless quantitity using the parameter $E_{0}$, the same field parameter used by Schäffer et al., ${ }^{7}$ and the measured initial characteristic time from each curve, $\tau_{\text {meas }}$, was similarly reduced to a dimensionless characteristic time using the characteristic time scale, $\tau_{0}$.

$$
\begin{gathered}
E_{\mathrm{P}}=\frac{U}{\epsilon_{\mathrm{P}} d-\left(\epsilon_{\mathrm{P}}-1\right) h_{0}} \\
\lambda=2 \pi \sqrt{\frac{E_{0}=\frac{U}{\lambda_{0}}}{\epsilon_{0} \epsilon_{\mathrm{P}}\left(\epsilon_{\mathrm{P}}-1\right)^{2}}} E_{\mathrm{P}}^{-3 / 2} \\
\tau=\frac{3 \gamma \eta}{\epsilon_{0}^{2} h_{0}^{3} U^{4}}\left(\frac{\epsilon_{0} \epsilon_{\mathrm{P}}\left(\epsilon_{\mathrm{P}}-1\right)^{2} U^{2}}{\gamma} \epsilon_{\mathrm{P}}-1-h_{0}\right)^{6}\left(1-\frac{1}{\epsilon_{\mathrm{P}}}\right)^{2} \\
\tau_{0}=\frac{3 \gamma \eta U^{8}}{\pi^{4} \epsilon_{0}{ }^{2} h_{0}^{3}}\left(\frac{\epsilon_{0} \epsilon_{\mathrm{P}}\left(\epsilon_{\mathrm{P}}-1\right)}{\gamma}\right)^{6}\left(1-\frac{1}{\epsilon_{\mathrm{P}}}\right)^{2} \\
\frac{\tau}{\tau_{0}}=\pi^{4}\left(\frac{E_{\mathrm{P}}}{E_{0}}\right)^{-6}
\end{gathered}
$$

In the above expressions, $\gamma$ is the polymer surface tension, $U$ is the applied voltage, $\eta$ is the viscosity, $h_{0}$ is the initial polymer film thickness, $d$ is the separation distance between the two electrodes, $\epsilon_{0}$ is the permittivity in a vacuum, and $\epsilon_{\mathrm{P}}$ is the polymer dielectric constant. These experimental parameters are used to calculate $E_{\mathrm{P}}$, the electric field strength in the polymer, and $E_{0}, \lambda_{0}$, and $\tau_{0}$, the characteristic parameters used to reduce experimental results to dimensionless values. Equations 1-4 and 7 were taken from ref 7 , whereas eqs 5 and 6 were derived from the analysis therein. Equation 7 shows the expected relationship between the dimensionless values.

A log-log plot of $\tau / \tau_{0}$ as a function of $E_{\mathrm{P}} / E_{0}$ is shown in Figure 4, along with predicted values according to eqs 5 and 6 . No fitting parameters were used to scale the data. The error bars in the figure are due to the uncertainties in the exponential fit. The scatter in the data, though, may arise from the uncertainty with which the fastest growing wavelength is chosen from within the field of view in the microscope. It is not possible to know which peak corresponds precisely to the fastest growing wave in the system. Although the growth rate of the first peak to reach the upper electrode is measured, we do not have sufficient resolution to obtain a statistical measurement of the growth rate of all peaks. We note that the values and trends of the experimental results, over 4 orders of magnitude in $\tau / \tau_{0}$, correspond remarkably well (within a factor of 2) to the predicted values for the three samples of PDMS with different viscosities that were studied. No systematic deviations from the predicted characteristic time values were seen as a function of viscosity. To within experimental errors a power law with an exponent of -6 was 
seen in the experimental data as would be expected theoretically. Thus, if experiments are restricted to the very early stages of growth in the surface fluctuations, quantitative agreement between experiment and theory can be obtained. The accelerated growth at later times will, of course, give rise to marked departures from the linear approximation and, as such, pronounced disagreement with linearized predictions.

\section{Conclusions}

The evolution of electric field-induced instabilities in thin polymer films was shown to be characterized by an exponential growth in peak height with time at early times. At later stages of growth, an acceleration in the growth rate with time was observed. The characteristic time for growth during the early stages agrees very well with theoretical predictions using a simple linear instability analysis. Surface waves having a characteristic periodicity grew simultaneously across the film surface, in a manner akin to spinodal phase separation in the early stages. Experiments in progress assess the changes in the shapes of the fluctuations prior to the formation of columns that span the gap to the upper electrode. In addition, by chemical modification of the surfaces of the film and electrode, the reversibility of the process is being examined. Finally, although the agreement of these measurements with theoretical predictions is excellent, methods with more statistical certainty, such as small-angle light scattering or off-specular X-ray reflectivity, are being pursued to provide confirmation of the microscope-based experiments.

Acknowledgment. This work was funded by NASA under Contract NAG8-694, the National Science Foundation supported Materials Research Science and Engineering Center at the University of Massachusetts (DMR98-09365), and the Department of Energy, Office of Energy Sciences (DE-FG-96ER45612). The authors also acknowledge insightful discussions with U. Steiner and E. Schäffer.

\section{References and Notes}

(1) See, for example: Vrij, A. Discuss. Faraday Soc. 1966, 423, 23; Annu. Rev. Fluid Mech. 1979, 11, 371. deGennes, P. G. Rev. Mod. Phys. 1985, 57, 827. Dussan, V. E.; BrochardWyart, F.; Daillant, J. Can. J. Phys. 1990, 68, 4-1088. Reiter, G. Phys. Rev. Lett. 1992, 68, 75-78. Brochard-Wyart, F.; Daillant, J. Langmuir 1993, 9, 2-3690. Jacobs, K.; Herminghaus, S.; Mecke, K. Langmuir 1998, 14, 965-969. Sharma, A.; Khanna, R. Phys. Rev. Lett. 1998, 81, 3463-3466. Kerle, T.; Yerushalmi-Rozen, R.; Klein, J.; Fetters, L. J. Europhys. Lett. 1998, 44, 484-490.

(2) Swan, J. W. Proc. R. Soc. London 1897, 62, 38-46.

(3) Doshi, J.; Reneker, D. H. J. Electrostat. 1995, 35, 151-160. Yarin, A. L.; Koombhongse, S.; Reneker, D. H. J. Appl. Phys. 2001, 90, 4836-4846.

(4) Shin, Y. M.; Hohman, M. M.; Brenner, M. P.; Rutledge, G. C. Polymer 2001, 42, 9955-9967. Shin, Y. M.; Hohman, M. M.; Brenner, M. P.; Rutledge, G. C. Appl. Phys. Lett. 2001, $78,1149-1151$.

(5) Oddershede, L.; Nagel, S. Phys. Rev. Lett. 2000, 85, 6.

(6) Schäffer, E.; Thurn-Albrecht, T.; Russell, T. P.; Steiner, U. Nature (London) 2000, 403, 874-877.

(7) Schäffer, E.; Thurn-Albrecht, T.; Russell, T. P.; Steiner, U. Europhys. Lett. 2001, 53, 518-524.

(8) Chou, S. Y.; Zhuang, L. J. Vac. Sci. Technol., B 1999, 17, 3197-3202.

(9) Chou, S. Y.; Zhuang, L.; Guo, L. Appl. Phys. Lett. 1999, 75, 1004-1006.

(10) Deshpande, P.; Sun, X.; Chou, S. Y. Appl. Phys. Lett. 2001, $79,1688-1690$

(11) Lin, Z.; Kerle, T.; Baker, S. M.; Hoagland, D. A.; Schäffer, E.; Steiner, U. Russell, T. P. J. Chem. Phys. 2001, 114, 23772381.

(12) Lin, Z.; Kerle, T.; Russell, T. P.; Schäffer, E.; Steiner, U. Macromolecules 2002, 35, 3971-3976.

(13) Pease, L. F., III.; Russell, W. B. Langmuir 2004, 20, 795.

(14) Lin, Z. Doctoral Dissertation, University of Massachusetts, 2003.

(15) Schaffer, E.; Harkema, S.; Blossey, R.; Steiner, U. Europhys. Lett. 2002, 60, 255.

MA048157P 